J. Austral. Math. Soc. 19 (Series B), (1976), 478-492.

\title{
CONTROL SYSTEMS WITH TIME LAGS
}

\author{
JOHN M. BLATT
}

(Received 14 January 1976)

\begin{abstract}
Necessary conditions for optimal controls are given for non-linear systems with time delayed effects in both control and state variables.
\end{abstract}

\section{Introduction}

Time-delayed optimal control problems have been considered by many authors. [1-11] The time-delayed terms may be restricted to state variables. $[1,2,3,5]$ Other authors have allowed time-delayed effects of control variables, but the control variables may enter only linearly. $[4,7]$

Non-linear time-delayed control and state variables have been considered by N. D. Georganas. $[11,12]$ Our present work is closely related to his.

To elucidate the differences, consider a simple special case:

(i) Only one, scalar, state variable $x(t)$.

(ii) Only one, scalar, control variable $v(t)$.

(iii) Only one, constant, time delay $\theta>0$.

(iv) A fixed planning horizon $T$, with no condition on $x(T)$.

The control problem involves three functions, $f(x, v, t), g(x, v, t)$, and $f_{0}(x, v, t)$, where $x$ is real, $v$ is within some control set $\Omega$, and $0 \leqq t \leqq T$. All these functions are taken to be differentiable with respect to $x$, continuous in $v$, and piecewise continuous in $t$ with at most a finite number of points of discontinuity. We want to extremize

$$
J=\int_{0}^{T} f_{0}[x(t), v(t), t] d t
$$

subject to 


$$
\begin{gathered}
\frac{d x}{d t}=f[x(t), v(t), t] \text { for } 0<t \leqq \theta \\
=f[x(t), v(t), t]+g[x(t-\theta), v(t-\theta), t-\theta] \text { for } \theta<t \leqq T \\
x(0)=a=\text { given }
\end{gathered}
$$

$v(t)$ piecewise continuous, $v(t) \in \Omega$ for all $0 \leqq t \leqq T$

Problems of this type arise in many areas. For example, $x(t)$ may be a population at time $t, v(t)$ the birth-rate (considered controllable), $f_{0}(x, v, t)$ some kind of instantaneous utility associated with this population, birth-rate, and time; the meaning of the time delay $\theta$ is then the lifetime of each member of the population (considered to be exactly the same for all). The differential equation which governs the time dependence of the population $x(t)$ is

$$
\frac{d x}{d t}=x(t) v(t)-x(t-\theta) v(t-\theta) \quad \text { for } \quad \theta<t \leqq T
$$

Comparison with (1.2b) allows us to identify $f(x, v, t)=x v$ and $g(x, v, t)=$ - $x v$. Georganas $[11,12]$ writes the same equation (1.4) in the form:

$$
\frac{d x}{d t}=A_{0}(t) f[x(t), v(t), t]+A_{1}(t) f[x(t-\theta), v(t-\theta), t-\theta]
$$

where

$$
f(x, v, t)=x v, \quad A_{0}(t)=1, \quad A_{1}(t)=-1
$$

The differences between (1.2) and (1.5) are:

(a) In (1.2), $f$ and $g$ may have different functional dependence on all three variables $x, v, t$. In (1.5), however, the instantaneous and time-delayed terms have the same functional dependence on two of these three variables, namely $x$ and $v$. This can be a serious restriction in practice.

(b) More important, there is a basic conceptual difference. Georganas assumes that his equation (1.5) holds for all $t$ in $0 \leqq t \leqq T$. This includes values of $t$ for which the precursor time $t^{\prime}=t-\theta$ is negative. He is therefore forced to consider controls $v\left(t^{\prime}\right)$ at negative times, $-\theta<t^{\prime}<0$; and he must state "initial conditions" on $x\left(t^{\prime}\right)$ over that same time interval, rather than merely at the point $t=0$. In his later paper [12] he introduces the concept of "penalization of the initial data", i.e., the cost functional is taken to depend on the values of the adjustable control $v\left(t^{\prime}\right)$, and of the given "initial data" $\phi\left(t^{\prime}\right)$ (equal to $x\left(t^{\prime}\right)$ in the interval $-\theta<t^{\prime}<0$ ) at negative times.

In our view, the past is beyond control. It is given history and should be treated as such. Thus, we do not assume that (1.2b) holds for times $t$ such that 
$t^{\prime}=t-\theta$ is negative. For those times, we use equation (1.2a) instead. In our simple example, let us denote the actual number of births per unit time by $b(t)$

$$
b(t)=x(t) v(t)
$$

We consider $b(t)$ controllable for $t>0$, but we insist that it is a given function of $t$ for $t \leqq 0$, completcly beyond control. Thus (1.4) is not valid for $t \leqq \theta$, and must be replaced by

$$
\frac{d x}{d t}=x(t) v(t)-\beta(t) \quad \text { for } \quad 0<t \leqq \theta
$$

where $\beta(t) \equiv b(t-\theta)$ is a given function of $t$. This is the conceptual difference between Georganas' approach and ours.

As a result of this conceptual difference, there are several differences in the mathematical formulation. These include:

(i) Our initial condition (1.3a) is at a point $(t=0)$, not over an interval $(-\theta<t<0)$.

(ii) The differential equation (1.2) assumes different forms in different time intervals, see (1.2a) and (1.2b).

(iii) A control function $v(t)$ is defined only on $0 \leqq t \leqq T$; no control exists at negative times.

(iv) The function $f(x, v, t)$ cannot be restricted to be continuous, but must be allowed to have discontinuities at isolated points (see below).

This last point (iv) emerges from our example. If we compare (1.6) with (1.2a), and (1.4) with (1.2b), we get

$$
\begin{aligned}
& f(x, v, t)=x v-\beta(t) \quad \text { for } \quad 0<t \leqq \theta \\
& =x v \text { for } \theta<t \leqq T \\
& g(x, v, t)=-x v \quad \text { for } \quad 0<t \leqq T-\theta
\end{aligned}
$$

Thus $f(x, v, t)$ is clearly a discontinuous function of $t$ at $t=\theta$, although it is differentiable in $x$ and continuous in $v$ for any given $t$ (we assume left-hand continuity in $t$ at all points of discontinuity, so that $f$ is defined also at $t=\theta$ ).

Although the usual proof techniques [11,12] depend on continuity, it turns out that a finite number of points of discontinuity cause no difficulty.

We note that there is an asymmetry between our equations (1.1) and (1.2): (1.2) contains time-delayed terms whereas (1.1) does not. (The same asymmetry occurs between equations (1) and (2) of Georganas. [12]) We wish to point out that this does not result in any significant loss of generality.

To see this, consider the apparently more general cost functional: 


$$
\hat{J}=\int_{0}^{T} \hat{f}_{0}[x(t), v(t), t] d t+\int_{\theta}^{T} \hat{g}_{0}[x(t-\theta), v(t-\theta), t-\theta] d t
$$

where $\hat{f}_{0}(x, v, t)$ and $\hat{g}_{0}(x, v, t)$ are given functions. We assert that $J$, far from being more general than (1.1), can be rewritten in the form (1.1). Just introduce $t^{\prime}=t-\theta$ in the second integral of (1.8) to get

$$
\hat{J}=\int_{0}^{T} \hat{f}_{0}[x(t), v(t), t] d t+\int_{0}^{T-\theta} \hat{g}_{0}\left[x\left(t^{\prime}\right), v\left(t^{\prime}\right), t^{\prime}\right] d t^{\prime}
$$

This is of the form (1.1), with

$$
f_{0}(x, v, t)=\hat{f}_{0}(x, v, t)+e(T-\theta-t) \hat{g}_{0}(x, v, t)
$$

where $e(\tau)$ is the unit step function defined by

$$
\begin{aligned}
& e(\tau)=0 \quad \text { for } \quad \tau \leqq 0 \\
& =1 \text { for } \tau>0
\end{aligned}
$$

Since isolated discontinuities in $f_{0}(\cdot, \cdot, t)$ are permissible, expression (1.9) for $f_{0}$ is quite acceptable. Thus the extra complication of (1.8) over (1.1) is not justified by any gain in generality.

Section 2 is devoted to a simple, heuristic derivation of a Pontryagin-type maximum principle for the control problem (1.1)-(1.3). The derivation is along the lines of chapter 4 of Hadley and Kemp, [13] and we do not attempt, in this paper, to make the argument rigorous (although this can be done). Rather, Teo and Moore [14] give a rigorous argument using the conventional techniques of control theory introduced by Pontryagin; we refer the reader to their paper for a rigorous proof. Section 3 of our paper is devoted to various extensions of the theory beyond the extremely simplified problem (1.1)-(1.3).

\section{Heuristic derivation of a maximum principle}

We rewrite (1.2) as a single equation by using the step function $e(t)$, (1.10):

$$
\frac{d x}{d t}=f[x(t), v(t), t]+e(t-\theta) g[x(t-\theta), v(t-\theta), t-\theta]
$$

We also define $x_{0}(t)$ by

$$
x_{0}(t)=\int_{0}^{\prime} f_{0}\left[x\left(t^{\prime \prime}\right), v\left(t^{\prime \prime}\right), t^{\prime \prime}\right] d t^{\prime \prime}
$$

so that $x_{0}(t)$ satisfies 


$$
\begin{gathered}
\frac{d x_{0}}{d t}=f_{0}[x(t), v(t), t] \\
x_{0}(0)=0
\end{gathered}
$$

The quantity to be optimized, (1.1), equals $x_{0}(T)$. For definiteness, we shall take our optimum to mean a maximum.

Assume that an optimal control $v^{*}(t)$ exists, is piecewise continuous as a function of $t$, and that $x^{*}(t)$ is the corresponding optimal path. We then have

$$
\frac{d x^{*}}{d t}=f\left[x^{*}(t), v^{*}(t), t\right]+e(t-\theta) g\left[x^{*}(t-\theta), v^{*}(t-\theta), t-\theta\right]
$$

Following standard procedure, we introduce a varied control $v(t)$. Let $\varepsilon>0$ and $\tau$ be such that $0<\tau \leqq T, 0 \leqq \tau-\varepsilon<T$, and the interval $\tau-\varepsilon \leqq t<\tau$ contains no discontinuity of $f, g, f_{0}$, or $v^{*}$. Let $v$ be any member of the control set $\Omega$. Then

$$
\begin{aligned}
v(t) & =v & & \text { for } \tau-\varepsilon<t \leqq \tau \\
& =v^{*}(t) & & \text { for all other } t \text { in }[0, T]
\end{aligned}
$$

The resulting path $x(t)$ satisfies (2.1) and (1.3a). For small enough $\varepsilon$, it turns out $[13,14]$ that $x(t)$ is related to $x *(t)$ by

$$
x(t)=x^{*}(t)+\varepsilon \zeta(t)+o(\varepsilon)
$$

and clearly

$$
\zeta(t)=0 \quad \text { for } \quad 0 \leqq t \leqq \tau-\varepsilon
$$

The difference between $v(t)$ and $v^{*}(t)$ enters the right-hand side of $(2: 1)$, and hence alters $d x / d t$, within $t w o$ distinct time intervals

(i) $\tau-\varepsilon<t \leqq \tau$

(ii) $\tau+\theta-\varepsilon<t \leqq \tau+\theta$

In the first of these intervals, the instantaneous term $f(x, v, t)$ in (2.1) differs from its optimal value. In the second time interval, the delayed term $g$ has $v(t-\theta)$ different from $v^{*}(t-\theta)$, because the precursor time $t^{\prime}=t-\theta$ falls within the range $\tau-\varepsilon<t^{\prime} \leqq \tau$.

In both these special time intervals, the function $\zeta(t)$ in (2.6) changes very rapidly. For time interval (i) one obtains, with conventional methods

$$
\begin{aligned}
x(\tau) & -x^{*}(\tau)=\varepsilon \zeta(\tau)+o(\varepsilon) \\
& =\int_{\tau-*}^{\tau}\left\{f\left[x\left(t^{\prime \prime}\right), v, t^{\prime \prime}\right]-f\left[x^{*}\left(t^{\prime \prime}\right), v^{*}\left(t^{\prime \prime}\right), t^{\prime \prime}\right]\right\} d t^{\prime \prime} \\
& =\varepsilon\left\{f\left[x^{*}(\tau), v, \tau\right]-f\left[x^{*}(\tau), v^{*}(\tau), \tau\right]\right\}+o(\varepsilon)
\end{aligned}
$$


so that

$$
\zeta(\tau)=\zeta(\tau)-\zeta(\tau-\varepsilon)=f\left[x^{* *}(\tau), v, \tau\right]-f\left[x^{*}(\tau), v^{*}(\tau), \tau\right]
$$

In this result, it is not necessary that $f(\cdot, \cdot, t)$ be continuous at $t=\tau$, only that it and $v^{*}(t)$ be left-hand continuous at $t=\tau$ and continuous in $\tau-\varepsilon \leqq t<\tau$.

In the second region, $\tau+\theta-\varepsilon<t \leqq \tau+\theta$, the relevant term in (2.1) is the delayed term, $g$. An entirely similar argument leads to

$$
\zeta(\tau+\theta)-\zeta(\tau+\theta-\varepsilon)=g\left[x^{*}(\tau), v, \tau\right]-g\left[x^{*}(\tau), v^{*}(\tau), \tau\right]+o(\varepsilon)
$$

At all other times $t$ in $\tau<t \leqq T$, we have $v(t)=v^{*}(t)$ and $v(t-\theta)=$ $v^{*}(t-\theta)$. We subtract (2.4) from (2.1) and use (2.6) to get

$$
\begin{aligned}
\frac{d \zeta}{d t}= & \frac{1}{\varepsilon}\left\{f\left[x^{*}(t)+\varepsilon \zeta(t), v^{*}(t), t\right]-f\left[x^{*}(t), v^{*}(t), t\right]\right\} \\
+ & e(t-\theta) \frac{1}{\varepsilon}\left\{g\left[x^{*}(t-\theta)+\varepsilon \zeta(t-\theta), v^{*}(t-\theta), t-\theta\right]\right. \\
& \left.-g\left[x^{*}(t-\theta), v^{*}(t-\theta), t-\theta\right]\right\}+o(1)
\end{aligned}
$$

Since $\partial f / \partial x$ and $\partial g / \partial x$ exist by assumption, the mean-value theorem of the differential calculus can be used to simplify this expression. A more detailed argument [14] shows that

$$
\frac{d \zeta}{d t}=\frac{\partial f}{\partial x} \zeta(t)+e(t-\theta)\left(\frac{\partial g}{\partial x} \zeta\right)_{t-\theta}+o(1)
$$

where $\partial f / \partial x$ is evaluated at $\left(x^{*}(t), v^{*}(t), t\right)$ and $(\partial g / \partial x) \zeta$ is evaluated at $\left(x^{*}(t-\theta), v^{*}(t-\theta), t-\theta\right)$. Equation (2.10) holds within the following time intervals:

(i) $0<t \leqq \tau-\varepsilon$ (trivially, $\zeta=0$ and $e(t-\theta)=0$ )

(ii) $\tau<t \leqq \min (\tau+\theta-\varepsilon, T)$

(iii) if $T>\tau+\theta$, in $\tau+\theta<t \leqq T$

For the remaining time intervals, $\tau-\varepsilon<t \leqq \tau$ and $\tau+\theta-\varepsilon<t \leqq \tau+\theta$, we use (2.8) and (2.9), respectively.

The variable $x_{0}(t),(2.2)$, satisfies the differential equation (2.3) and can thus be treated similarly. Its optimum path is denoted by $x_{0}^{*}(t)$ and is related to the varied path $x_{0}(t)$ by

$$
x_{0}(t)=x_{0}^{*}(t)+\varepsilon \zeta_{0}(t)+o(\varepsilon)
$$

with

$$
\zeta_{0}(t)=0 \text { for } 0 \leqq t \leqq \tau-\varepsilon
$$




$$
\begin{gathered}
\zeta_{0}(\tau)=f_{0}\left[x^{*}(\tau), v, \tau\right]-f_{0}\left[x^{*}(\tau), v^{*}(\tau), \tau\right] \\
\frac{d \zeta_{0}}{d t}=\frac{\partial f_{0}}{\partial x} \zeta \quad \text { for } \quad \tau<t \leqq T
\end{gathered}
$$

where $\partial f_{0} / \partial x$ in $(2.12 \mathrm{c})$ is to be evaluated at the point $\left(x^{*}(t), v^{*}(t), t\right)$. Equations (2.12) are simpler than the corresponding equations for $\zeta(t)$, because by assumption (2.3a) contains no time-delayed terms (see the discussion in section 1. after equation (1.8)). Note that the right-hand side of (2.12c) contains $\zeta(t)$, not $\zeta_{0}(t)$.

Since $x_{0}^{*}(T)$ is an optimum (i.e., a maximum) by assumption, we have $x_{0}(T) \leqq x_{0}^{*}(T)$, so that (2.11) together with $\varepsilon>0$ yields

$$
\zeta_{0}(T) \leqq 0
$$

At this stage, we need to define a Hamiltonian for this problem. It is best to use the time-integrated functional

$$
\mathscr{H}=\int_{0}^{T}\left(\lambda_{0} \frac{d x_{0}}{d t}+\lambda(t) \frac{d x}{d t}\right) d t
$$

where we are to substitute the right-hand sides of (2.1) and (2.3a) for $d x / d t$ and $d x_{0} / d t$, respectively. Straightforward substitution gives

$$
\begin{aligned}
\mathscr{H}=\int_{0}^{T} & \left\{\lambda_{0} f_{0}(x, v, t)+\lambda(t) f(x, v, t)\right. \\
& \quad+e(t-\theta) \lambda(t) g[x(t-\theta), v(t-\theta), t-\theta]\} d t .
\end{aligned}
$$

However, there is also an equivalent form, obtained by introducing the dummy variable $t^{\prime}=t-\theta$ in the last term

$$
\begin{aligned}
\mathscr{H}=\int_{0}^{T} & \left\{\lambda_{0} f_{0}(x, v, t)+\lambda(t) f(x, v, t)\right\} d t \\
& +\int_{0}^{T-\theta} \lambda\left(t^{\prime}+\theta\right) g\left[x\left(t^{\prime}\right), v\left(t^{\prime}\right), t^{\prime}\right] d t^{\prime}
\end{aligned}
$$

The canonical equations $d x / d t=\delta \mathscr{H} / \delta \lambda(t)$ and $d x_{0} / d t=\delta \mathscr{H} / \delta \lambda_{0}(t)$ are reduced to explicit form most easily by starting from (2.14) for $\mathscr{H}$. These equations are just (2.1) and (2.3a), respectively.

The adjoint equations for the co-state variables, on the other hand, are derived most easily by starting from (2.15). They turn out to be 


$$
\frac{d \lambda}{d t}=-\frac{\delta \mathscr{H}}{\delta x(t)}=-\lambda_{0} \frac{\partial f_{0}}{\partial x}-\lambda(t) \frac{\partial f}{\partial x}-e(T-\theta-t) \lambda(t+\theta) \frac{\partial g}{\partial x}
$$

where $\partial f_{0} / \partial x, \partial f / \partial x$, and $\partial g / \partial x$ are to be evaluated at $(x(t), v(t), t)$; and

$$
\frac{d \lambda_{0}}{d t}=-\frac{\delta \mathscr{H}}{\delta x_{0}(t)}=0 .
$$

Thus $\lambda_{0}(t)$ is a constant, and the differential equation (2.16) for $\lambda(t)$ contains, in the last term on the right-hand side, the value of $\lambda$ at the later time $t^{\prime \prime}=t+\theta$, provided $t^{\prime \prime}$ is not beyond the planning horizon $T$ (in that case, the unit step function $e(T-\theta-t)$ becomes zero).

It is relevant to remark that the differential equation for the state variable $x(t)$, equation (2.1), and for the co-state variable $\lambda(t)$, equation (2.16), both allow systematic numerical integration, but from opposite directions: To find $x(t)$ from (2.1), we must integrate forward in time, for only then is $x(t-\theta)$ a known quantity; to find $v(t)$ from (2.16), we must integrate in the direction of decreasing time $t$, i.e., backwards from $t=T$, for only then is $\lambda(t+\theta)$ a known quantity.

Let us now define $Z(t)$ by

$$
Z(t)=\lambda_{0} \zeta_{0}(t)+\lambda(t) \zeta(t) .
$$

In order to relate $Z(T)$ to the inequality (2.13), we impose the conditions:

$$
\lambda(T)=0 \quad \lambda_{0}=1, \quad \text { all } t
$$

the first of which acts as an initial condition on $\lambda(t)$ if we integrate (2.16) backwards from $t=T$. (2.13), (2.18) and (2.19) give

$$
Z(T)=\zeta_{0}(T) \leqq 0
$$

In ordinary control theory, $Z(t)$ turns out to be a constant of motion, i.e., $d Z / d t=0$. This is not true in time-delayed control theory. However, we assert that the following equality holds:

$$
\begin{aligned}
Z(T)=Z(\tau) & +e(T-\tau-\theta) \lambda(\tau+\theta)\left\{g\left[x^{*}(\tau), v, \tau\right]-g\left[x^{*}(\tau), v^{*}(\tau), \tau\right]\right\} \\
& +o(1)
\end{aligned}
$$

To prove this, we write

$$
Z(T)-Z(\tau)=\int_{\tau}^{\tau+\theta-\varepsilon} \frac{d Z}{d t} d t+Z(\tau+\theta)-Z(\tau+\theta-\varepsilon)+\int_{\tau+\theta}^{\tau} \frac{d Z}{d t} d t .
$$

We now use (2.10), (2.12c), (2.16), (2.17) and (2.18) to simplify the two integrals to get 


$$
\begin{aligned}
Z(T)-Z(\tau)= & \left(\int_{\tau}^{\tau+\theta-\sigma} d t+\int_{\tau+\theta}^{\tau} d t\right)\left\{e(t-\theta) \lambda(t)\left(\frac{\partial g}{\partial x} \zeta\right)_{t-\theta}\right. \\
& \left.-e(T-\theta-t) \lambda(t+\theta)\left(\frac{\partial g}{\partial x} \zeta\right)\right\} \\
& +\zeta_{0}(\tau+\theta)-\zeta_{0}(\tau+\theta-\varepsilon) \\
& +\{\lambda(\tau+\theta) \zeta(\tau+\theta)-\lambda(\tau+\theta-\varepsilon) \zeta(\tau+\theta-\varepsilon)\}
\end{aligned}
$$

In the first line on the right-hand side, the additional interval from $\tau+\theta-\varepsilon$ to $\tau+\theta$ contributes an amount of higher order for small $\varepsilon$; and in the last term, replacing $\lambda(\tau+\theta-\varepsilon)$ by $(\tau+\theta)$ also contributes an amount of higher order in $\varepsilon$, since the differential equation (2.16) is quite well-behaved for $\tau+\theta-\varepsilon<t \leqq \tau+\theta$. Using (2.9) and the fact that $\zeta_{0}(t)$ does not jump sharply in $\tau-\theta-\varepsilon<t \leqq \tau+\theta$, we therefore obtain

$$
\begin{aligned}
Z(T)-Z(\tau)= & \int_{\tau}^{T}\left\{e(t-\theta) \lambda(t)\left(\frac{\partial g}{\partial x} \zeta\right)_{,-\theta}-e(T-\theta-t) \lambda(t+\theta)\left(\frac{\partial g}{\partial x} \zeta\right)_{t}\right\} d t \\
& +\lambda(\tau+\theta)\left\{g\left[x^{*}(\tau), v, \tau\right]-g\left[x^{*}(\tau), v^{*}(\tau), \tau\right]\right\}+o(1) .
\end{aligned}
$$

The last term is what we want for (2.21). The extra factor $e(T-\tau-\theta)$ makes explicit what is already understood in (2.22) and earlier, namely

$$
\lambda(t)=0 \quad \text { for } \quad t \geqq T .
$$

The point is quite simple: An "advanced effect" at time $t$ " $=t+\theta$ can enter the equations only if $t^{\prime \prime}$ is still within the planning period, $t^{\prime \prime}<T$. In writing our formulas, leading to (2.22), we have assumed tacitly that $\tau+\theta<$ $T$. This tacit assumption underlies (2.22), and in (2.21) the factor $e(T-\tau-\theta)$ makes the assumption explicit: this factor vanishes unless $\tau+\theta<T$. The formal expression (2.23) is consistent with (2.16) and (2.19), i.e., $\lambda(t)=0$ for all $t \geqq T$ is a solution of that system.

It remains to show that the integral in (2.22) vanishes. This is a difference of two integrals. Because of (2.7) we can write the first integral as

$$
I_{1}=\int_{t+\theta}^{T} \lambda(t)\left(\frac{\partial g}{\partial x} \zeta\right)_{t-\theta} d t
$$

(i.e., there is no contribution from $\tau \leqq t \leqq \tau+\theta$ since $\zeta(t-\theta)$ vanishes there). Similarly, the factor $e(T-\theta-t)$ allows us to write the second integral as

$$
I_{2}=\int_{\tau}^{\tau-\theta} \lambda(t+\theta)\left(\frac{\partial g}{\partial x} \zeta\right)_{1} d t
$$


However, a simple change of dummy variable, $t^{\prime}=t-\theta$, converts the integral $I_{1}$ into the form $I_{2}$, so that $I_{1}=I_{2}$ and thus $I_{1}-I_{2}=0$.

Q.E.D.

Now substitute (2.18) for $Z(\tau)$ into (2.21), and use (2.20) for the left-hand side. This gives the inequality

$$
\begin{aligned}
\lambda_{0} \zeta_{0}(\tau) & +\lambda(\tau) \zeta(\tau)+e(T-\tau-\theta) \lambda(\tau+\theta)\left\{g\left[x^{*}(\tau), v, \tau\right]\right. \\
& \left.-g\left[x^{*}(\tau), v^{*}(\tau), \tau\right]\right\} \leqq 0 .
\end{aligned}
$$

Since $\lambda_{0}=1, \zeta_{0}(\tau)$ is given by $(2.12 \mathrm{~b})$ and $\zeta(\tau)$ by $(2.8),(2.24)$ is expressible entirely in terms of known quantities.

In order to express the inequality (2.24) succinctly, we now define the point Hamiltonian $H\left(\lambda_{0}, \lambda, x, v, t\right)$ by

$$
\begin{aligned}
H\left(\lambda_{0}, \lambda, x, v, t\right) \equiv & \lambda_{0} f_{0}(x, v, t)+\lambda(t) f(x, v, t) \\
& +e(T-\theta-t) \lambda(t+\theta) g(x, v, t) .
\end{aligned}
$$

A look at (2.15) shows that this definition is consistent with the known value of the integrated Hamiltonian $\mathscr{H}$, i.e., it follows from (2.15) that.

$$
\mathscr{H}=\int_{0}^{T} H\left(\lambda_{0}, \lambda, x, v, t\right) d t .
$$

However, we emphasize that (2.25) is not a consequence of (2.26). We used the integrand of (2.15) for our definition (2.25); but as far as satisfying (2.26) is concerned, we might just as well have used the integrand of (2.14), with the same result for $\mathscr{H}$ but a quite different form for $H$. The real reason for choosing (2.25) is not (2.26), but rather the fact that we wish to simplify the inequality (2.24). This inequality reduces very nicely if and only if we define $H$ by (2.25); then (2.24) reduces to the maximum principle

$$
H\left[\lambda_{0}, \lambda(\tau), x^{*}(\tau), v^{*}(\tau), \tau\right] \geqq H\left[\lambda_{0}, \lambda(\tau), x^{*}(\tau), v, \tau\right]
$$

for all $v \in \Omega$, the control set. That is, the optimal control $v^{*}(\tau)$ at each time $\tau$, $0<\tau \leqq T$, is such as to make the Hamiltonian (2.25) a maximum with respect to all permitted values of $v$. (2.27) is the desired extension of the Pontryagin maximum principle to the time-delayed control problem.

We note that $H$ has a different functional form for different regions of $t$. When (2.25) is written out explicitly, it gives

$$
\begin{aligned}
& H=\lambda_{0} f_{0}(x, v, t)+\lambda(t) f(x, v, t)+\lambda(t+\theta) g(x, v, t) \\
& \text { for } 0<t \leqq T-\theta \\
& H=\lambda_{0} f_{0}(x, v, t)+\lambda(t) f(x, v, t) \text { for } T-\theta<t \leqq T .
\end{aligned}
$$


There is no reason to expect the value of $H$ to be independent of the time $t$, or even to be a continuous function of $t$. On the contrary, one should expect a discontinuity at $t=T-\theta$.

Note that (2.29) is the ordinary Pontryagin Hamiltonian for a problem without time-delays. Since (2.27) tells us to maximize $H$, we conclude that the time-delayed terms do not directly influence the choice of the optimal control $v^{*}(t)$ for $T-\theta<t \leqq T$. This makes sense intuitively: For $T-\theta<t \leqq T$, a time-delayed effect, occurring at $t^{\prime \prime}=t+\theta$, is not visible within our planning horizon (i.e., $t^{\prime \prime}>T$ ), and can therefore have no influence on maximization of (1.1).

This argument, however, implies a warning against uncritical application of this theory! In many, indeed in most, practical situations a sharply defined planning horizon $T$ is only a rough idealization of the true problem. An "optimization" which ignores all effects occurring at times $t>T$ may be of very doubtful value.

One way out is an infinite planning horizon, coupled with discounting of the future. Let $U(x, v)$ be some "utility" of the state $(x, v)$ at time $t$, and let $\rho>0$ be a discount rate. Then we may use the theory to maximize

$$
J=\lim _{T \rightarrow \infty} \int_{0}^{T} \exp (-\rho t) U[x(t), v(t)] d t
$$

subject to (2.2) and to $v(t) \in \Omega$, all $t \geqq 0$. If the limit exists, the effect is to make the region $T-\theta<t \leqq T$ unimportant, since it shifts out to infinity and its effect is discounted by an exponential factor no larger than $\exp [-\rho(T-\theta)]$, which goes to zero. In this limit, therefore, we expect (2.28) to remain relevant for all finite values of $t$.

\section{Extensions of the theory}

(a) VECTORS:

Let the state $x(t)$ be a vector with $n$ components

$$
x(t)=\left(x_{1}(t), x_{2}(t), \ldots, x_{n}(t)\right) .
$$

Similarly, $v(t)$ may be a vector with $m$ components ( $m$ may differ from $n$ ). We still wish to maximize (1.1), but the differential equation (1.2), or (2.1), now is replaced by the coupled set:

$$
\begin{gathered}
\frac{d x_{1}}{d t}=f_{\mathrm{i}}[x(t), v(t), t]+e(t-\theta) g_{\mathrm{t}}[x(t-\theta), v(t-\theta), t-\theta] \\
i=1,2, \ldots, n .
\end{gathered}
$$


This reduces to (2.1) for $n=1$. In (1.3b), $\Omega$ is a subset of an $m$-dimensional vector space. The co-state variable $\lambda$ becomes an $n$-vector also, and (2.16) is replaced by the coupled set

$$
\begin{aligned}
\frac{d \lambda_{t}}{d t}= & -\lambda_{0} \frac{\partial f_{0}}{\partial x_{i}}-\sum_{i=1}^{n} \lambda_{l}(t) \frac{\partial f_{j}}{\partial x_{i}} \\
& -e(T-\theta-t) \sum_{i=1}^{n} \lambda_{l}(t+\theta) \frac{\partial g_{i}}{\partial x_{i}} \quad i=1,2, \ldots, n .
\end{aligned}
$$

This reduces to (2.16) for $n=1$. Finally, the Hamiltonian is defined by

$$
\begin{aligned}
H\left(\lambda_{0}, \lambda, x, v, t\right) & =\lambda_{0} f_{0}(x, v, t)+\sum_{i=1}^{n} \lambda_{i}(t) f_{j}(x, v, t) \\
& +e(T-\theta-t) \sum_{j=1}^{n} \lambda_{i}(t+\theta) g_{i}(x, v, t) .
\end{aligned}
$$

This reduces to (2.25) for $n=1$.

The maximum principle, in the form (2.27), is unaltered, and so are the differential equations (2.3a) for $x_{0}$ and (2.17) for $\lambda_{0}$. If $v$ is a vector control variable, then both $v^{*}$ and $v$ in (2.27) are vectors, and are to be varied over the permitted (vector) control set $\Omega$, a subset of the full $m$-dimensional vector space $R^{m}$.

There is no substantial change in the argument of section 2 , and the trivial modifications are left as an exercise to the reader.

(b) Multiple time delays:

In many practical applications, there are several time-delayed terms, with different delay times. We denote the delay times by $\theta_{1}, \theta_{2}, \cdots, \theta_{s}$, with

$$
0<\theta_{1}<\theta_{2}<\cdots<\theta_{s} \text {. }
$$

The differential equations (3.1) are then extended to

$$
\begin{gathered}
\frac{d x_{i}}{d t}=f_{i}(x(t), v(t), t)+\sum_{\alpha=1}^{s} e\left(t-\theta_{\alpha}\right) g_{i \alpha}\left[x\left(t-\theta_{\alpha}\right), v\left(t-\theta_{\alpha}\right), t-\theta_{\alpha}\right] \\
i=1,2, \cdots, n .
\end{gathered}
$$

This reduces to (3.1) when $s=1$. The adjoint equations (3.2) become:

$$
\begin{gathered}
\frac{d \lambda_{i}}{d t}=-\lambda_{0} \frac{\partial f_{0}}{\partial x_{i}}-\sum_{j=1}^{n} \lambda_{l}(t) \frac{\partial f_{j}}{\partial x_{i}} \\
-\sum_{\alpha=1}^{s} e\left(T-\theta_{\alpha}-t\right) \sum_{i=1}^{n} \lambda_{j}\left(t+\theta_{\alpha}\right) \frac{\partial g_{j a}}{\partial x_{i}} \\
i=1,2, \cdots, n .
\end{gathered}
$$


The Hamiltonian is:

$$
\begin{aligned}
H\left(\lambda_{0}, \lambda, x, v, t\right) & =\lambda_{0} f_{0}(x, v, t)+\sum_{j=1}^{n} \lambda_{j}(t) f_{j}(x, v, t) \\
& +\sum_{\alpha=1}^{s} e\left(T-\theta_{\alpha}-t\right) \sum_{j=1}^{n} \lambda_{j}\left(t+\theta_{\alpha}\right) g_{j \alpha}(x, v, t) .
\end{aligned}
$$

The derivation of the maximum condition (2.27) is made more awkward by the presence of jumps in $\zeta(t)$ at a number of times $t^{\prime}>\tau$, namely at $t^{\prime}=\tau+\theta_{1}, t^{\prime}=\tau+\theta_{2}, \cdots, t^{\prime}=\tau+\theta_{s}$ (or rather as many of those as are no greater than $T$ ). But this is a mere complication of detail, involving no new essential point.

(c) Continuously distributed time delays:

New points do arise in connection with time-delays which are distributed continuously, i.e., instead of discrete delays $\theta_{1}, \cdots, \theta_{s}$ as in (3.4) we have a continuous variable $\theta$. We do not attempt, in this paper, to discuss this problem in proper mathematical fashion; but we do wish to motivate, by means of a heuristic argument, a conjecture concerning the following control problem.

Let $f_{0}(x, v, t), f_{1}(x, v, t)$, and $h_{t}(x, v, t, \theta)$ for $i=1,2, \cdots, n$ be given functions. The problem is to maximize

$$
x_{0}(T)=\int_{0}^{T} f_{0}[x(t), v(t), t] d t
$$

subject to $(i=1,2, \cdots, n)$

$$
\begin{aligned}
& \frac{d x_{i}}{d t}=f_{i}[x(t), v(t), t]+\int_{0}^{t} h_{i}\left[x\left(t^{\prime}\right), v\left(t^{\prime}\right), t^{\prime}, t-t^{\prime}\right] d t^{\prime} \\
& x_{1}(0)=a_{1} \text { given } \\
& v(t) \in \Omega \text { (given control set), for all } 0 \leqq t \leqq T .
\end{aligned}
$$

Note that the $h_{t}$ are explicitly functions of $\theta=t-t^{\prime}$, and also that the precursor time $t^{\prime}$ in (3.9) is never negative, nor can it ever exceed the current time $t$ : We neither attempt to control the past (negative times), nor do we permit effects at time $t$ to be due to causes in the future $\left(t^{\prime}>t\right)$.

In order to motivate our conjecture, we rewrite (3.9) in terms of the time delay $\theta=t-t^{\prime}$, and we let $\theta$ go from 0 to $T$, formally, so as to have a fixed interval of integration:

$$
\begin{aligned}
\frac{d x_{i}}{d t}= & f_{i}[x(t), v(t), t] \\
& +\int_{0}^{T} e(t-\theta) h_{i}[x(t-\theta), v(t-\theta), t-\theta, \theta] d \theta
\end{aligned}
$$


Now divide the interval $(0, T)$ into a large number $s$ of sub-intervals of equal length. We define

$$
\theta_{\alpha}=\alpha(T / s) \quad \alpha=1,2, \cdots, s
$$

and we approximate the integral in (3.12) by a sum to get

$$
\begin{aligned}
\frac{d x_{1}}{d t}= & f_{i}[x(t), v(t), t] \\
& +\sum_{\alpha=1}^{s} e\left(t-\theta_{\alpha}\right) g_{1 \alpha}\left[x\left(t-\theta_{\alpha}\right), v\left(t-\theta_{\alpha}\right), t-\theta_{\alpha}\right]
\end{aligned}
$$

where

$$
g_{i \alpha}[x, v, t]=(T / s) h_{1}\left[x, v, t, \theta_{\alpha}\right] .
$$

The approximate form (3.14) of (3.9) is identical with (3.5). Our conjecture consists in a transliteration of equations (3.6) and (3.7) to the integral form, i.e., a step inverse to the one which we made to get (3.14) from (3.9). The resulting equations for the co-state variables $\lambda_{1}(t)$ are (see (3.6)):

$$
\begin{aligned}
\frac{d \lambda_{i}}{d t}= & -\lambda_{0} \frac{\partial f_{0}}{\partial x_{i}}-\sum_{j=1}^{n} \lambda_{j}(t) \frac{\partial f_{i}}{\partial x_{i}} \\
& -\int_{0}^{T-t} \sum_{j=1}^{n} \lambda_{j}(t+\theta)\left(\frac{\partial h_{i}}{\partial x_{i}}\right) d \theta
\end{aligned}
$$

where $\partial h_{1} / \partial x_{t}$ in the last term is evaluated at $x(t), v(t), t, \theta$. The definition of the Hamiltonian is (see (3.7))

$$
\begin{aligned}
H\left(\lambda_{0}, \lambda, x, v, t\right)= & \lambda_{0} f_{0}(x, v, t)+\sum_{j=1}^{n} \lambda_{l}(t) f_{l}(x, v, t) \\
& +\int_{0}^{r-t} \sum_{i=1}^{n} \lambda_{l}(t+\theta) h_{l}[x(t), v(t), t, \theta] d \theta .
\end{aligned}
$$

Our conjecture is that a necessary condition for optimal control of problem (3.8)-(3.11) is the maximum condition (2.27), with $H$ defined by (3.17).

In conclusion, it is our pleasant duty to thank Professor M. Kemp and Dr. K. L. Teo for valuable discussions.

\section{References}

[1] L. S. Pontryagin, V. G. Boltyanskii, R. V. Gramkrelidze and E. F. Mishchenko, The Mathematical Theory of Optimal Processes, New York: Interscience Publishers, Inc., (1962); in particular, see pages $213 \mathrm{ff}$.

[2] Avner Friedman, 'Optimal Control for Hereditary Processes', Arch. Rational Mech. Anal. 15 (1964), 396.

[3] M. N. Oguztoreli, Time-Lag Control Systems, New York: Academic Press, (1966). 
[4] H. T. Banks, 'Necessary Conditions for Control Problems with Variable Time Lags', SIAM J. Control 6 (1968), 9-47.

[5] G. L. Kharatishvili, 'A Maximum Principle in Extremal Problems with Delays', Proc. Conf. on Math. Theory of Control, Univ. of South. Calif, edited by A. V. Balakrishnan and L. W. Neustadt, New York: Academic Press (1967), 26-34.

[6] A. Halanay, 'Optimal Controls for Systems with Time-Lag', SIAM J. Control 6 (1968), 215-235.

[7] E. B. Lee, 'Variational Problems for Sysiems having Delay in the Control Action', I.E.E.E. Trans. on Aut. Control (1968), 697-699.

[8] E. B. Lee and L. Markus, Foundations of Optimal Control Theory, New York: John Wiley (1967).

[9] K. S. Day and T. C. Hsia, 'Optimal Control of Linear Time-Lag Systems', Proc. J.A.C.C., The University of Michigan (1968), 1046-1055.

[10] D. H. Chyung and E. B. Lee, Optimal Systems with Time Delays, Proc. 3rd I.F.A.C. Conf., London (1966).

[11] N. D. Georganas, Optimal Control for a Class of Hereditary Systems, Ph.D. thesis, Dept. of Elec. Eng., University of Ottawa (1970).

[12] N. D. Georganas, "Optimization in a class of hereditary systems with direct penalization of the initial data", Int. J. Systems Sci. 5 (1974), 295-299.

[13] G. Hadley and M. C. Kemp, Variational Methods in Economics, Amsterdam: NorthHolland Publishing Coy. (1971).

[14] K. L. Teo and E. J. Moore, 'Necessary Conditions for Optimality for Control Problems with Time Delays Appearıng in Both State and Control Variables', to be published.

Department of Applied Mathematics,

University of New South Wales,

Kensington,

Australia. 